\title{
Testing Astrophysical Radiation Hydrodynamics Codes with Hypervelocity Jet Experiments on the Nova Laser
}

\author{
J. M. Stone, N. Turner
}

This article was submitted to Second International Workshop on Laboratory Astrophysics with Intense Lasers, Tucson, AZ, March 19-21, 1999

U.S. Department of Energy

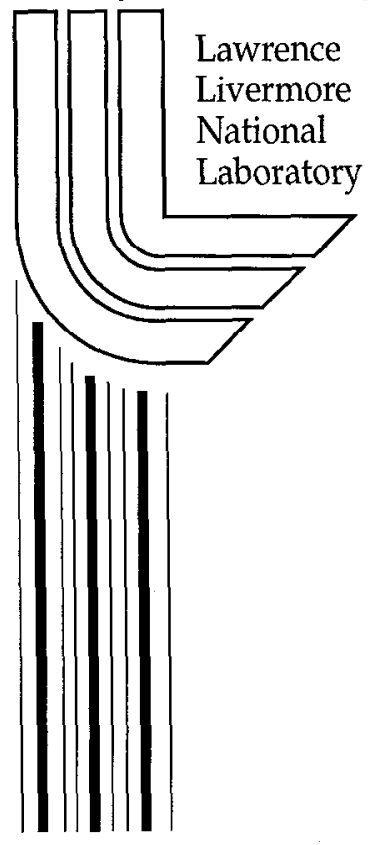

September 22, 1999 


\section{DISCLAIMER}

This document was prepared as an account of work sponsored by an agency of the United States Government. Neither the United States Government nor the University of California nor any of their employees, makes any warranty, express or implied, or assumes any legal liability or responsibility for the accuracy, completeness, or usefulness of any information, apparatus, product, or process disclosed, or represents that its use would not infringe privately owned rights. Reference herein to any specific commercial product, process, or service by trade name, trademark, manufacturer, or otherwise, does not necessarily constitute or imply its endorsement, recommendation, or favoring by the United States Government or the University of California. The views and opinions of authors expressed herein do not necessarily state or reflect those of the United States Government or the University of California, and shall not be used for advertising or product endorsement purposes.

This is a preprint of a paper intended for publication in a journal or proceedings. Since changes may be made before publication, this preprint is made available with the understanding that it will not be cited or reproduced without the permission of the author.

This report has been reproduced directly from the best available copy.

Available to DOE and DOE contractors from the Office of Scientific and Technical Information

P.O. Box 62, Oak Ridge, TN 37831

Prices available from (423) 576-8401

http://apollo.osti.gov/bridge/

Available to the public from the National Technical Information Service

U.S. Department of Commerce 5285 Port Royal Rd., Springfield, VA 22161 http://www.ntis.gov/

OR

Lawrence Livermore National Laboratory Technical Information Department's Digital Library http://www.llnl.gov/tid/Library.html 
Testing Astrophysical Radiation Hydrodynamics Codes with Hypervelocity Jet Experiments on the Nova Laser

\author{
James M. Stone, Neal Turner \\ Department of Astronomy \\ University of Maryland \\ College Park, MD 20742-2421
}

and

Kent Estabrook, Bruce Remington, David Farley, S. Gail Glendinning'

Lawrence Livermore National Laboratory, L-021, Livernore, CA 94550

Received __ _ accepted _ _ _ 


\begin{abstract}
Recent shock tube experiments using the Nova laser facility have demonstrated that strong shocks and highly supersonic flows similar to those encountered in astrophysical jets can be studied in detail through carefully controlled experiment. We propose the use of high power lasers such as Nova, Omega, and NIF to perform experiments on radiation hydrodynamic problems. such as jets involving the multidimensional dynamics of strong shocks. High power lasers are the only experimental facilities that can reach the very high Mach number regime. The experiments will serve both as diagnostics of astrophysically interesting gas dynamic problems, and could also form the basis of test problems for numerical algorithms for astrophysical radiation hydrodynamic codes. The potential for experimentally achieving a strongly radiative jet seems very good.
\end{abstract}




\section{Introduction}

Numerical methods are an important tool for studying gas dynarnical problems in astrophysics. Before becoming useful, however, such methods must be calibrated through a careful testing process. Such testing is designed not only to uncover simple errors in the implementation, but also serves to demonstrate the strengths and limitations of a given method on a wide variety of problems.

Testing of astrophysical gas dynamic codes usually employs one of three techniques: (1) comparison of numerical calculations with the results of laboratory experiments, (2) comparison of numerical results with analytic solutions where they exist, and (3) cross comparison of numerical solutions generated by different algorithms. An example of the first technique for hydrodynamical algorithms is provided by the classic Sod shocktube problem (Sod 1978), which was originally motivated by experiments, and is now universally adopted. In fact, the Sod shocktube problem has an analytic solution, and it has been used to test nearly every numerical method for compressible hydrodynamics, so that it also may be regarded as an example of the second and third techniques of testing. Collections of teat problems which include examples of each type have been published for both hydrodynamic (e.g. Sod 1978; Woodward \& Colella 1994) and magnetohydrodynamic (e.g. Stone et al 1992; Toth \& Odstrcil 1996) codes.

Radiation hydrodynamic codes are not as commonly used as hydrodynamic or MHD codes in astrophysics, despite their importance to the study of problems ranging from winds from hot stars, accretion disks around compact objects and active galactic nuclei, and strong shocks. Moreover, finding test problems for such codes is difficult due to the challenge of finding analytic solutions to the dynamical equations, and the lack of published results from other codes with which to compare. There is an obvious lack of published test 
problems for radiation hydrodynamic codes, compared to the case for hydrodynamic or MHD codes

The availability of results from laboratory experiments which study radiation hydrodynamic problems would benefit the development, testing, and ultimately the application of numerical methods for astrophysical problems, just as experiments contributed to the development and testing of hydrodynamic and MHD methods. In this short communication, we describe several experiments which are currently underway on. the Nova laser facility at the Lawrence Livermore National Laboratory to study radiation hydrodynamic problems such as hypervelocity jets. The hope is that these types of data can eventually be used to test numerical codes.

\section{Proposed Nova Experiments}

There are three regimes in which the effects of radiation on the gas dynamics are important. The first is in the radiative cooling regime, i.e. optically thin gas which loses a substantial fraction of its internal energy through radiative losses. There are many examples of astrophysical flows in this regime; one which has been studied extensively is the propagation through the interstellar medium of supersonic jets from young stars (e.g., Blondin et al 1990; Stone \& Norman 1993, 1994; de Gouviea dal Pino \& Benz 1994), a simulation of which is shown in Fig. la. Since such jets are galactic, high resolution imaging and spectroscopic observations have revealed an enormous amount of detail which constrains dynamical models. A particularly good example is provided by the HH47 system (Heathcote et al 1996), located at a distance of $450 \mathrm{pc}$ in the Gum Nebula, and shown in Fig. 1b. The numerical hydrodynamic studies reported to date demonstrate that radiative cooling plays an essential role in the fragmentation and formation of structure in the 
outflowing jet material. The second regime is one in which momentum deposition from photons is important, but to a first approximation energy deposition is not. An example of such flows is the dynamics of winds from hat stars (Owocki 1994) or disks (Proga, Drew \& Stone 1998): radiative driving from spectral lines is fundamental to the flow, but to a good approximation the gas in the outflow can be considered as isothermal. Finally, the third regime is one in which both energy and momentum transport by photons is important, and the system must be considered as a fully coupled fluid composed of both particles and photons (Mihalas \& Mihalas 1984). The numerical techniques used to calculate the dynamics in each regime differ substantially. In the radiatively cooled regime, the dynamics can be captured simply through the addition of a loss term to the energy equation which can be computed from a standard optically thin cooling curve. In the momentum driven regime, a radiation force must be added to the equations of motion: computing this force in general is complex as it requires solution of the radiation transfer equation to account for optical depth effects. Finally, in the fully coupled regime, separate systems of conservation laws must be solved for both the material and radiation components of the plasma. Techniques for solving the hierarchy of radiation moment equations in multidimensions are complex and still emerging (e.g. Stone, Mihalas, \& Norman 1992).

Which regime a flow occupies depends on its density, temperature, and composition. It is likely that Nova laser experiments can be designed to study flows in the first (radiation cooling) and third (fully coupled) regimes. Moreover, lasers such as Nova, Omega, and NIF are the only experimental facilities capable of generating the very high Mach number fows relevant to astrophysical jets. The dynamics of jet evolution is qualitatively different at high Mach numbers (see Norman et al 1982), thus the need for experimental facilities that can reach the appropriate regime.

Experiments to study flows in the radiative cooling regime are being prepared, 
motivated by astrophysical studies of protostellar jets (see references listed above). Typical dimensionless parameters that characterize protostellar jets are internal Mach numbers $M \sim 10-40$, density ratios $\eta \equiv \rho_{\text {jet }} / \rho_{a m b i e n t}=1-10$, and radiative cooling parameters $\chi \equiv l_{\text {cool }} / R_{\text {jet }} \sim 0.2-2$. Here, $l_{\text {cool }}$ is the cooling length in either the shocked jet or ambient gas (if $\eta \neq 1$, the cooling length can be different in each region), and $R_{j e t}$ is the jet radius. For $\chi \gg 1$ the flow is effectively adiabatic, for $\chi \ll 1$ the flow is isothermal, while for $\chi \sim 1$ the characteristic radiative and hydrodynamic time scales are of the same order of magnitude. In this latter regime, the radiation and hydrodynamics are coupled. It is important to note that provided the dimensionless parameters of the flow established through a Nova experiment are the same as those of an astrophysical system, the results of the Nova experiment (which might extend for a fraction of a millimeter in size and last for only tens of nanoseconds) can be scaled to astrophysical jets (which extend for billions of kilometers and last for tens of thousands of years).

High Mach number $(M \sim 20)$, supersonic $\left(v_{j c t} \sim 60 \mathrm{~km} \mathrm{~s}^{-1}\right)$, adiabatic jets have already been studied by Miller et al (1995) and Logory et al (1999) on the Nova laser. A schematic of a typical experimental package is shown in Figure 2a. It consists of a brominated plastic ablator $\left(\rho_{\text {ablator }}=1.2 \mathrm{~g} \mathrm{~cm}^{-3}\right)$ followed by a foam tamper $\left(\rho_{\text {foam }}=0.1 . \mathrm{g} \mathrm{cm}^{-3}\right)$ At the ablator-foam interface, a $300 \mu \mathrm{m}$ diameter hemispherical notch was machined into the plastic. The experimental package was mounted on the side of a gold hohiraum (radiation cavity). Eight $1 \mathrm{~ns}$ Nova beams delivered a total of approximately $20 \mathrm{~kJ}$ of energy to the hohlraum, generating a thermal spectrum with characteristic temperature $T_{r}=220 \mathrm{eV} \mathrm{X}$-ray drive which ablatively launched a strong shock (with postshock pressure $\left.P_{\text {shock }} \sim 80 \mathrm{Mbar}\right)$ into the ablator. The material of the ablator near the vertex of the hemispherical notch races ahead into the foam upon passage of the shock, initiating the supersonic jet, which was diagnosed by X-ray radiography. A typical radiograph taken at 
$\sim 40$ ns (Miller et al 1995) shows (Fig. 2b) a dense supersonic jet which has propagated about 2 jet radii from the hemisphere. The hydrodynamics of the jet has been modeled with the Livermore arbitrary Lagrange-Eulerian code CALE, as shown in Fig. 2c. In this simulation, CALE was run as a purely hydrodynamics code (radiation effects were not implemented). The goal of these experiments was to study the hydrodynamic aspects of supersonic, high Mach number jets, such as the jet tip speed and the Kelvin-Helmboltz rollups which can be observed in the experiment. However, the temperatures behind the bow shock, $T<30 \mathrm{eV}$, were not high enough for radiation to influence the ensuing hydrodynamics. These adiabatic jet experiments serve as a test of our ability to follow deep nonlinear, strong shock hydrodynamics with present astrophysical codes.

A variation on the experiment shown in Fig. 2 is illustrated in Fig. 3a. Here, the X-ray drive launches a shock into an aluminum "pin" (Foster et al 1998). The result is a high Mach number jet of $\mathrm{Al}$ traveling into the plastic (CH) receptacle, as shown with the data in Fig. 3b. One sees a beautiful Kelvin-Helmholtz rollup sitting just behind the bow shock. Due to the high Mach number $(M \sim 10)$ of the jet of $\mathrm{Al}$, the jet tip is in close proximity to the bow shock, which likely affects both the jet tip trajectory and also the shape of the jet. (Norman 1982; Holmes et al 1999).

We are now designing jet experiments that are intended to be radiative. We have considered four different approaches, as illustrated schematically in Figs. 4a-4d. In Fig. 4 a we show a design which is only a small change from that shown in Fig. 2. The simple modification is to form the $\mathrm{CH}(\mathrm{B}$.) ablator into a $\sim 100 \mu \mathrm{m}$ thick, $300 \mu \mathrm{m}$ diameter hemispherical shell. This new design launches a converging shock into the foam, which focuses compressed foam material onto the cylinder axis first, before launching the jet (of compressed foam) outwards through the uncompressed foam. This convergence raises the jet temperature considerably. The results from two-dimensional LASNEX (Zimmerman \& 
Kruer 1975) simulations of this proposed experiment suggest that localized temperatures near the jet axis can exceed $200 \mathrm{eV}$, nearly an order of magnitude higher than the adiabatic jet experiment of Miller et al (1995). Key parameters in the jet are consistent with those observed for astrophysical protostellar jets. Moreover, the LASNEX simulation shows that radiation has penetrated significantly ahead the bow shock. This radiative "preheat" warms the electron fluid, which in turn preionizes the plasma. This alters the propagation of the jet by increasing the sound speed and hence the velocity of the bow shock. This radiative preheat is easily understood from simple mean-free-path arguments. For $200 \mathrm{eV}$ photons radiating into $0.1 \mathrm{~g} \mathrm{~cm}^{-3}$ density carbon foam, the mean-free-path is approxinately $20 \mu \mathrm{m}$, and for $290 \mathrm{eV}$ photons, just below the $\mathrm{K}$ absorption edge of carbon, the mean-free-path is approximately $40 \mu \mathrm{m}$. These mean free paths are large enough to generate the radiative preheat penetration. Therefore, although the radiative cooling parameter $\chi$ for the Nova experiments may be higher than order 1 , as opposed to astrophysical jets where $\chi \sim 1$ there should be observable coupling between radiation and the hydrodynamics of the jet evolution. This raciative preionization (preheat) effect is also critical to an understanding of high velocity astrophysical protostellar jets. A slight modification to this design is illustrated in Fig. 4 b, where the hemisphere shape is replaced with a cone. The parameter regimes achieved are similar, i.e. peal temperatures of approximately $200 \mathrm{eV}$, with modest radiative effects.

In an attempt to generate hotter, more tenuous jets where the effects of radiation are more striking, we have considered the designs sketched in Figs. $4 \mathrm{c}$ and $4 \mathrm{~d}$. Here, the ablated plasma itself collides on axis, and forms the jet. Of the two cases considered, X-ray illumination and direct laser illumination, the latter generates the hotter jet with the more striking radiative effects, and is discussed next.

The direct laser illumination design uses the concave side of an iron cone, with full 
opening angle of 120 degrees. A short pulse of high intensity laser light is incident on the concave side of the Fe. The laser parameters used in the 2D LASNEX simulations are 1.5 $\mathrm{kJ}$ of $1 / 3 \mu \mathrm{m}$ wavelength light in a 100 ps Gaussian time profile, focused into a $600 \mu \mathrm{m}$ diameter spot. In Fig. 5a we show an isodensity contour plot from the simulations at $0.8 \mathrm{~ns}$. From the simulation, one sees a very focused, narrow jet at $z=400 \mu \mathrm{m}$ ernanates from the base of the cone. Due to the perfect symmetry in this $2 D$ simulation, the predicted width may be too narrow. Nevertheless, the simulations suggest that a well formed jet will emerge at a high velocity, about $700 \mu \mathrm{m} / \mathrm{ns}$ (ie, $700 \mathrm{~km} / \mathrm{s}$ ). To illustrate the effect of radiation in the jet hydrodynamics, we ran an identical simulation, only with radiation transport turned off. The equivalent isodensity contours at $0.8 \mathrm{~ns}$ are shown in Fig. $5 \mathrm{~b}$. One sees a dramatic change, with the jet now being very broad and diffuse (note the different scales). Without the ability to remove heat by radiative cooling, this purely hydrodynamic jet is very hot, and accordingly expands laterally. These comparisons are quantified in Figs. 5c and $5 \mathrm{~d}$, where we show the density and temperature profles as a function of lateral position (radius) at $z=400 \mu \mathrm{m}$ and $t=0.8 \mathrm{~ns}$, for the calculations with radiation "on" (solid curves) versus with radiation "off" (dashed curves). With radiation transport (and hence radiative cooling) included, the jet collapses down to a dense $\left(50-100 \mathrm{mg} / \mathrm{cm}^{3}\right)$, narrow ( $<50 \mu$ m diameter), relatively cool $(\sim 100 \mathrm{eV})$ jet traveling outwards along the $z$-direction at high velocity $(\sim 700 \mu \mathrm{m} / \mathrm{ns}=700 \mathrm{~km} / \mathrm{s})$. With radiation turned of in the simulation, the jet cannot radiate away its heat, so it "puffs up", thereby cooling itself by the $P(\nabla \cdot \mathbf{v})$ work of expansion. The width $(\sim 300 \mu \mathrm{m})$ and temperature $(\sim 750 \mathrm{eV})$, are nearly an order of magnitude larger, and the peak density on axis $\left(\sim 10 \mathrm{mg} / \mathrm{cm}^{3}\right)$ is nearly an order of magnitude lower in the non-radiative jet compared to its radiative counterpart. In addition, the much larger cross-sectional area of the non-radiative jet implies it will have a much larger mass per unit distance along its length as well. These effects should be dramatically 
apparent in the experiment. Even though the densities are much lower than the situation illustrated in Fig. 2, estimates of the ion-ion collision mean free path length suggest that the plasma is still collisional, that is, $\lambda_{i i} / w \ll 1$, where $w$ is a characteristic spatial scale of the problem. Estimates of the photon mean free path length for $\sim 1 \mathrm{keV}$ photons, however, suggest that the plasma is optically thin early in time ( $\leq 0.6 \mathrm{~ns})$ when the plasma is hot, that is, $\lambda_{m f p} / w \gg 1$.

We will experimentally diagnose the jet with side-on radiography using a $2 \mathrm{D}$ gated imager (Budil et al 1996) and with a uranium backlighter (Glendinning et al 1995). We will also do 2D gated imaging in emission, since the simulated jet is very hot and luminous, possibly 1D streaked spectroscopy, and Thompson scattering (Glenzer et al. 1998). The Thompson scattering and possibly emission spectroscopy should provide experimental measures of the electron temperature and densities, while the $2 D$ imaging will map out. the shape and trajectory of the jet. Initial experiments (Farley et al 1999), using the configuration shown in Fig, 4d, only with a Au instead of Fe cone, show that indeed a high velocity, luminous, narrow jet is formed, and quite likely is radiative. An example of a. static pinhole image in emission is shown in Fig. 6. The pinhole camera used 10 micron pinholes at $4 \mathrm{x}$ magnification, recorded directly onto DEF $\mathrm{X}$-ray file, and viewed the jet . from an angle of 68 degrees from the direction of propagation of the jet.

The above discussion serves as a design study; the actual laser experiments to study radiative hydrodynamic jets are just beginning. If successful, the experiments will provide detailed diagnostics of the resulting radiation-dominated flows, including two dimensional images from X-ray framing cameras at several snapshots in the evolution, and possibly kinematic information from X-ray spectroscopy in both emission and absorption (see Willi et al 1999). The experiments are interesting studies of radiation hydrodynamic flows in their own right. For example, the jet experiments designed as an extension of the 
work of Miller et al (1995) may provide useful information regarding the mixing rate in supersonic jets. In addition, however, the experiments can be used to provide the basis of a test suite of problems for radiation hydrodynamic codes used in astrophysics, much as the Richtmyer-Meshkov instability experiments of Dimonte et al (see Holmes et al 1999) performed on the Nova laser have become benchmark tests of strong shock dynamics for compressible hydrodynamic codes. By modeling the experiments with different numerical techniques, it may be possible to develop improvements, and explore the limitations, of individual methods. Such a test suite would be an extremely useful service to the progress of this research area within the astrophysico community.

Financial support of this worls from the DOE is gratefully acknowledged. We thank Larry Logory, and Paul Miller for helpful conversations. We are grateful to J. Foster, P. Rosen, and T. Perry for obtaining advance copies of their Al jet data prior to publication. This, work was performed under the auspices of the U.S. DOE by LLNL under contract No. W-7405-Eng-48. 


\section{REFERENCES}

Blondin, J.M., Fryxell, B.A., \& Konigl, A. 1990, Ap.J., 360, 370.

Budil et al.

Foster, J.M., et al. 1998, Bull. Am. Phys. Soc., 43, 1738 (Abstract G4F9)

Glendinning, S.G., et al 1995, in Applications of Laser Plasma Radiation II, SPIE Proceedings Vol. 2523 (SPIE-International Society for Optical Engineering, Bellingham, WA, p.29.

Glenzer, S.H., et al 1998, Phys. Rev. Lett., 82, 97.

de Gouvviea dal Pino, E.M., \& Benz, W. 1994, Ap.J., 435, 261

Heathcote, S. et al 1996, AJ, 112, 1141.

Holmes, R.L., et al, 199s9, J. Fluid Mech., 389, 55

Logory, L. et al, 1999, these proceedings.

Mihalas, D., \& Mihalas, B., 1984 Foundations of Radiation Hydrodynamics, (Oxford University Press, Oxford).

Miller, P.L., Peyser, T.A., Stry, P.E., \& Budil, K.S. 1995, in Shock Waves, eds. B. Sturtevant, J, Shepherd, and H. Hornung, (World Scientific, New Jersey, 1995), p. 581.

Norman, M.L., Winkler, K.-H, Smarr, L, \& Smith, M.D. 1982, A\&A, 113, 285.

Owocki, S. 1994, Ap.\& Sp. Sci, 221, 3.

Proga, D., Stone, J.M., \& Drew, J.E. 1998, MNRAS, 295, 59.5. 
Sod, G.A. 1978, J. Comp. Phys., 27, 1.

Stone, J.M., Hawley, J.F., Evans, C.R., \& Norman, M.L., 1992, Ap.J., 388, 415.

Stone, J.M., Mihalas, D., \& Norman, M.L., 1992, Ap.J.Supp., 80, 819.

Stone, J.M., \& Norman M.L. 1993, Ap.J., 413, 198

Stone, J.M., \& Norman M.L. 1994, Ap.J., 420, 237

Toth, G., \& Odstrcil, D. 1996, J. Comp: Phys., 128, 82.

Willi, O., et al, 1999, these proceedings.

Woodward, P.R., \& Colella, P., 1984, J. Comp. Phys., 54, 115.

Zimmerman, G.B., \& Kruer, W.L. 1975, Comm. Plasma Phys. Controlled Fusion, 2, 51.

\section{Figure Captions}

Figure 1. Examples of astrophysical jets. (a) Simulation of a Herbig-Haro jet, reproduced from (Stone \& Norman 1994). (b) Image of the Herbig-Haro jet HH47, a galactic astrophysical jet located in the Gum Nebula imaged with HST, reproduced from Heathcote et al (1996).

Figure 2. An example of a laboratory high Mach-number jet: (a) Experimental configuration for launching the hypervelocity jet, using the Nova laser. (b) Example of an in-fight, hard X-ray radiograph of the jet at $\sim 40 \mathrm{~ns}$, and (c) the corresponding result from 
a simulation with the $2 D$ radiation-hydrodynamics code CALE (see Logory et al.; these proceedings).

Figure 3. Another example of a laboratory hypersonic jet, generated on the Nova laser in a slightly different manner. (a) Experimental configuration for launching the jet, using the Nova laser. (b) Example of an in-flight radiograph of the jet at $\sim 15 \mathrm{~ns}$. The proximity of the Al jet tip to the bow shock in $\mathrm{CH}$ is a signature of high Mach number, $M \sim 10$ (see Foster et al 1998).

Figure 4. Examples of designs for a laboratory radiative jet experiment using the Nova laser. The first design (a) uses an $\mathrm{X}$-ray drive to launch a converging shock. The second design (b) uses the plasma colliding on axis after shock breakout, somewhat like a miniature shape charge. Designs (c) and (d) use the ablation plasma stagnating on axis from illumination with $X$-rays (c) and with direct laser illumination (d).

Figure 5. Results from design simulations for the experimental configuration shown in Fig. 4d. (a) Isodensity contours at $0.8 \mathrm{~ns}$, for the full radiative-hydrodynamics simulation. (b) The same as (a), only for a simulation with radiation turned off. (c) Latieral profile of density of the jet at $0.8 \mathrm{~ns}$ at a position of $z=400 \mu \mathrm{m}$, with radiation (solid curve) and without radiation (dashed curve) included in the simulation. (d) Same as (c) except for profiles of temperature.

Figure 6. An example of a static (i.e., time integrated) pinhole image in emission from a gold jet launched from the experimental configuration shown in Fig. $4 \mathrm{a}$. The pinhole camera used $10 \mu \mathrm{m}$ pinholes at $4 \mathrm{x}$ magnification, was filtered with $1 \mathrm{~mm}$ of Be, recorded directly onto DEF $X$-ray file, and viewed the jet from an angle of 68 degrees with respect to the direction of propagation. 


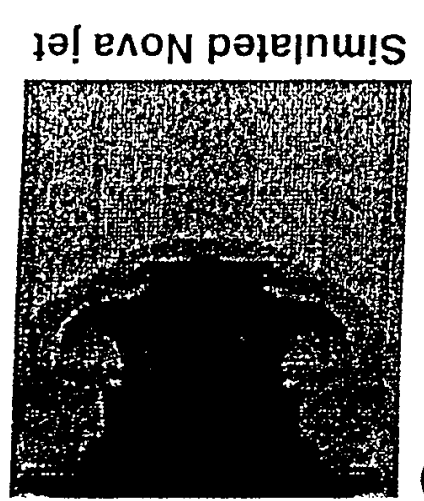

(0)

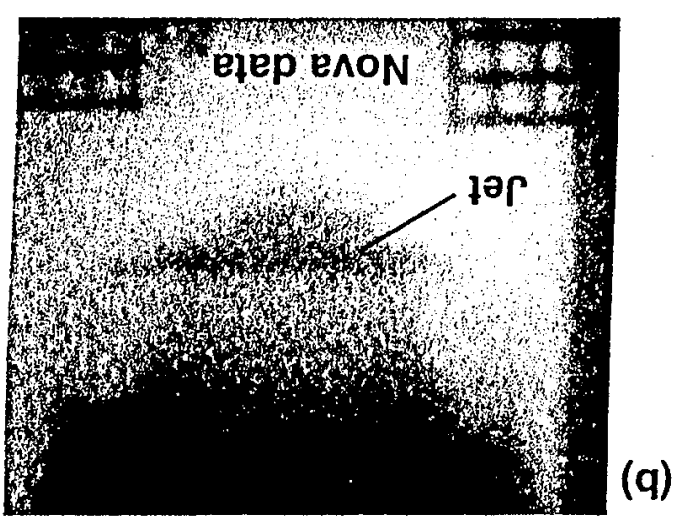

(q)

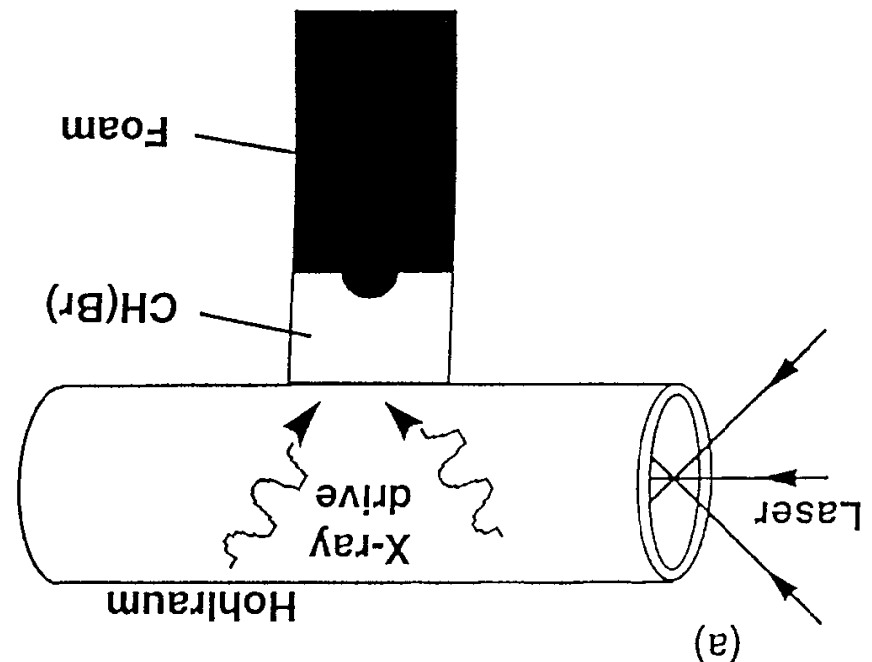

(e)

เ อมกต!ฺ
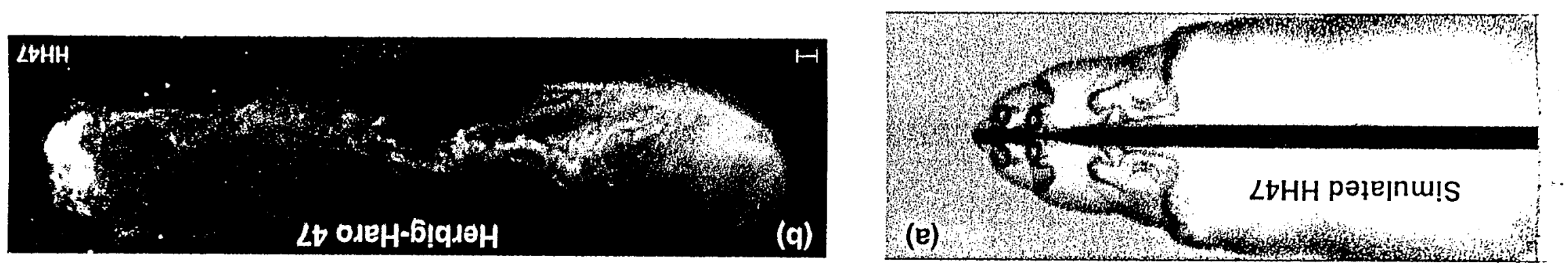
(a)

Hohlraum

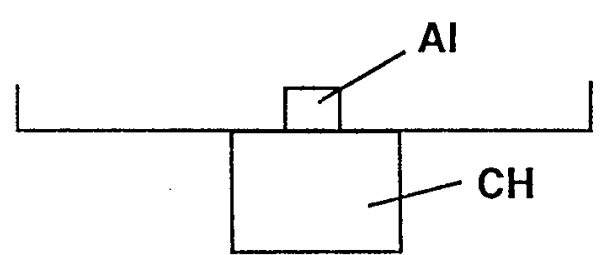

(b)

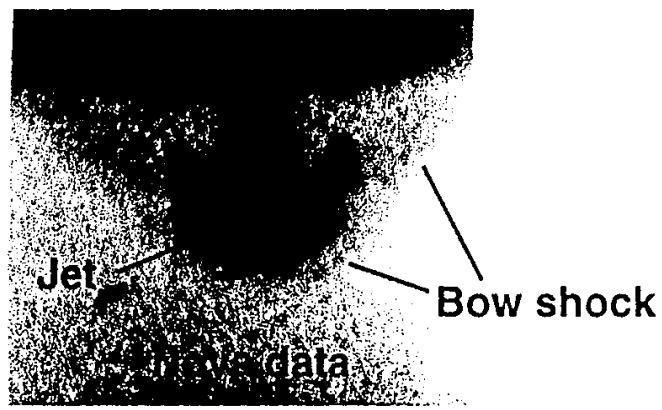

Figure 3

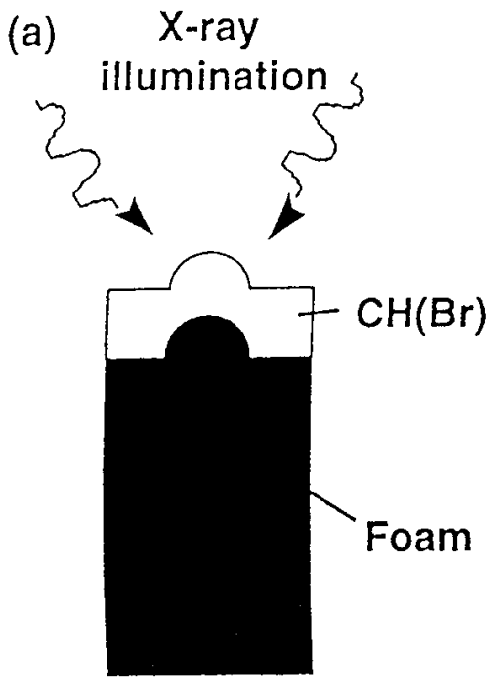

(b)

\section{X-ray} illumination

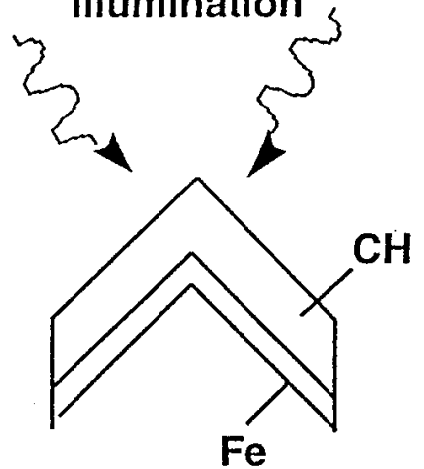

(c)

(d)

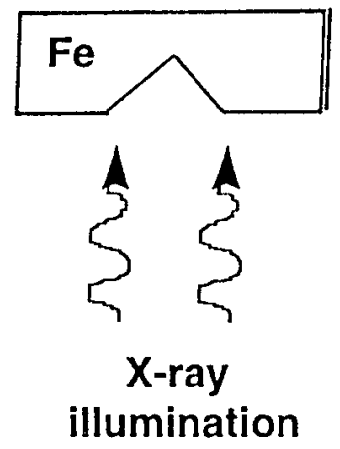

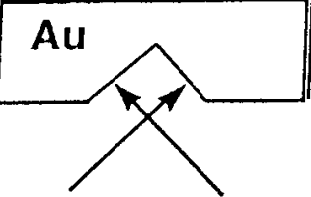

Direct laser illumination

Figure 4

Stone_Jet_Figures 3,4 
(a)

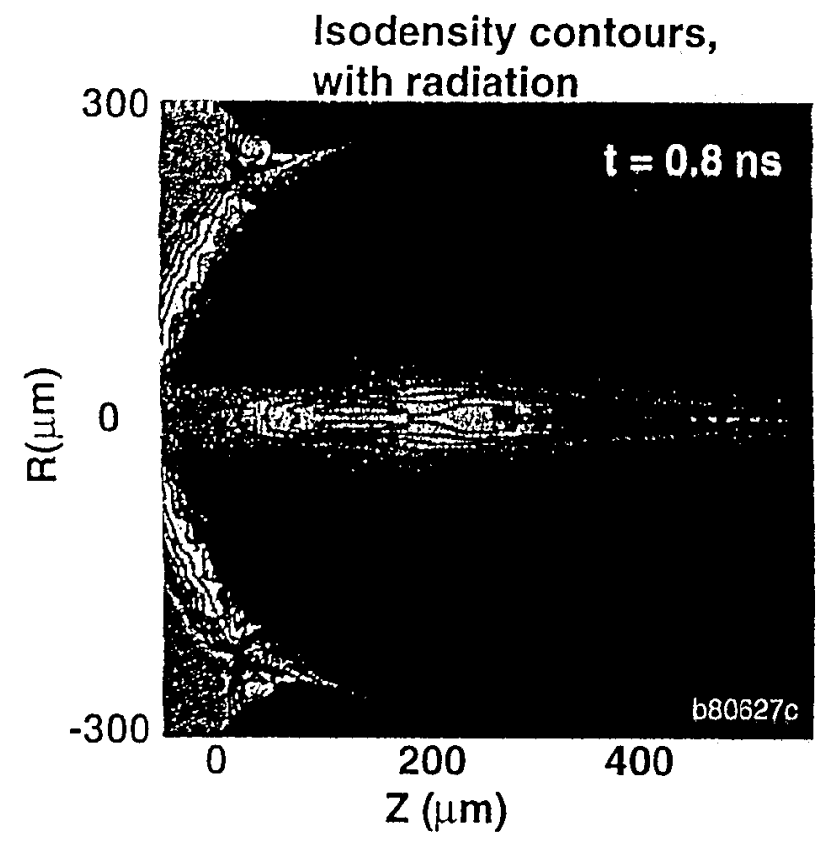

(c)

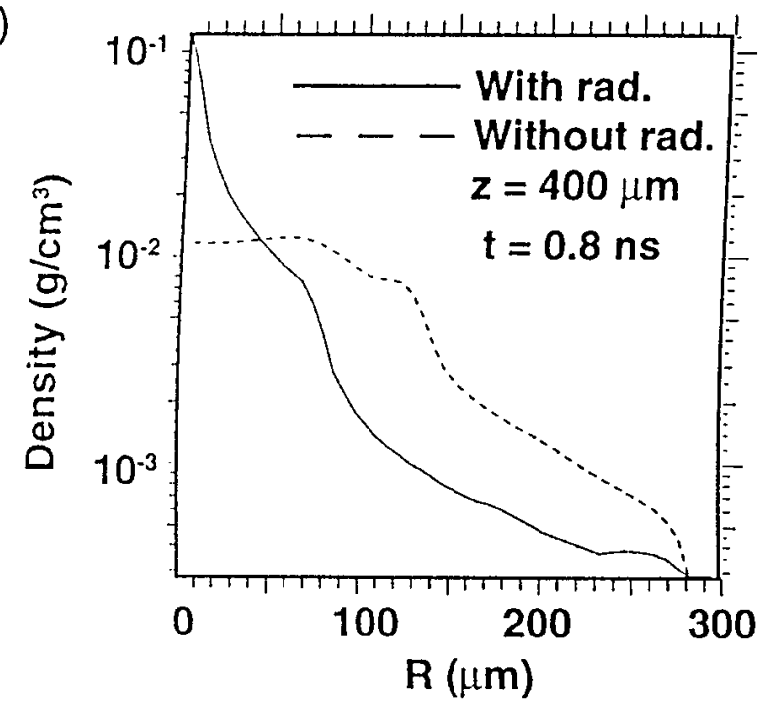

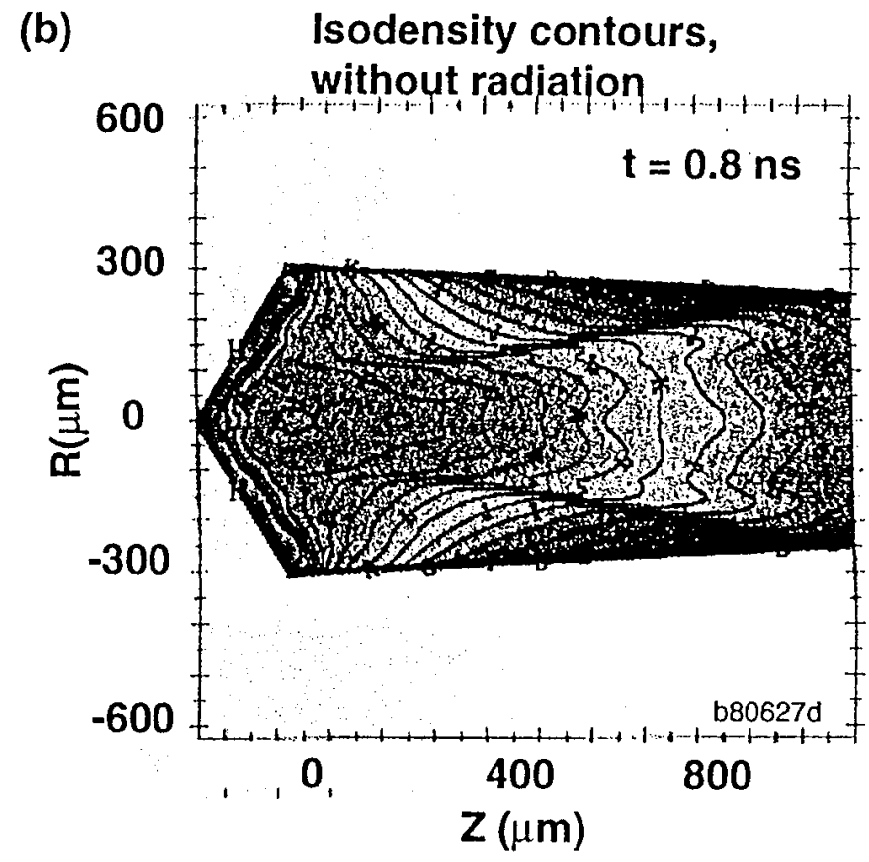

(d)

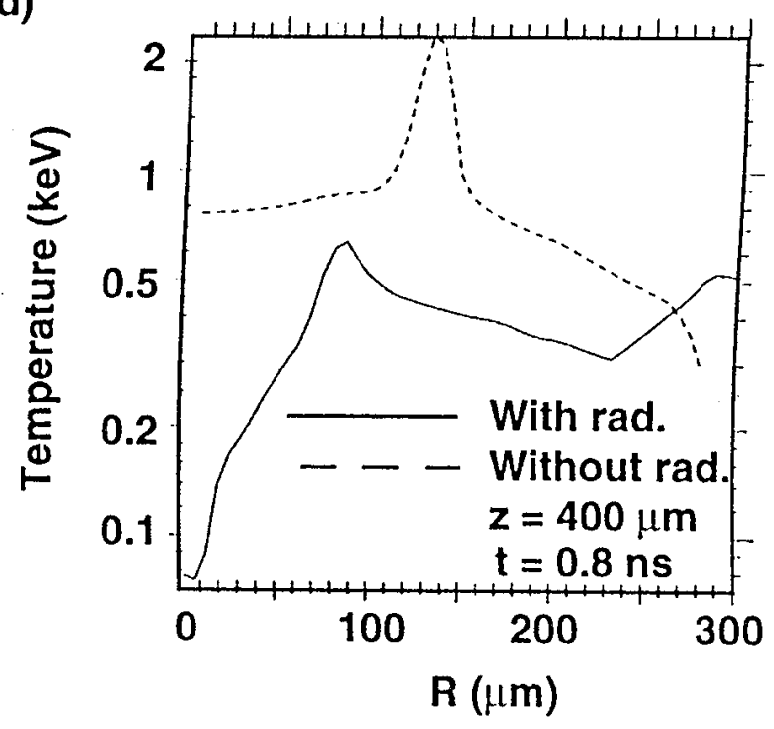

Figure 65 


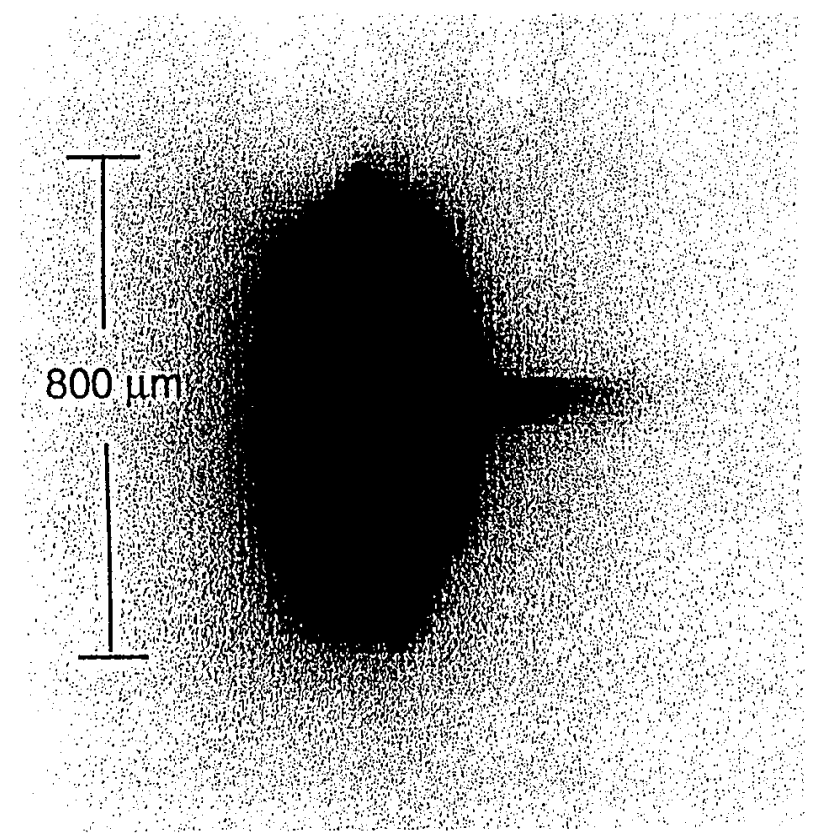

SPCA_1.TIFF

Stone_Jet_Fig. 7 : 